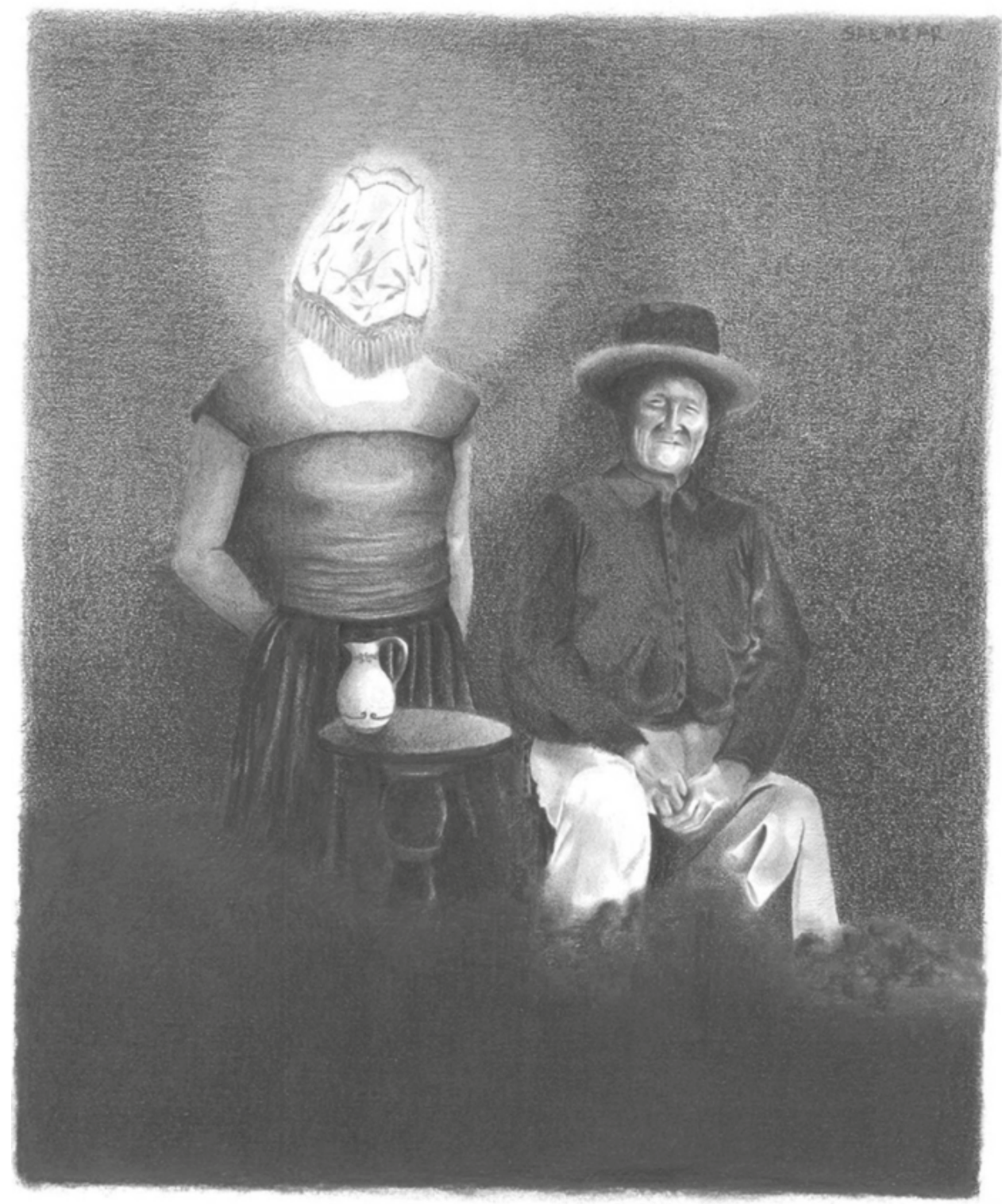

Luis Fernando Salazar Espinosa

Sin título

De la serie Psiquis

Lápiz sobre papel

$18 \times 15 \mathrm{~cm}$

2017

Medellín 


\title{
De línea en el desierto a espacio de sociabilidad. El discurso informativo de la prensa chilena sobre la frontera entre Chile y Perú (2014-2015)*
}

\author{
Daniel Bello Arellano (Chile) ${ }^{* *}$ \\ Magglio Gino Chiuminatto Orrego (Chile) ${ }^{* * * 0}$
}

\section{Resumen}

Este artículo analiza los discursos informativos sobre la frontera entre Chile y Perú publicados por la prensa chilena, con el objetivo de identificar y contrastar las diversas propuestas narrativas que intervienen en el proceso simbólico de construcción de la realidad fronteriza. De este modo se busca, por un lado, contribuir al estudio de un territorio tensionado por disputas bilaterales de larga data, constantemente creado y re-significado en y por los discursos públicos; por otro lado, comparar el rol de los medios nacionales y locales en dicho proceso creativo. El trabajo permitió identificar dos formas distintas de entender y narrar la frontera, que pueden sintetizarse en dos imágenes: la de una línea en el desierto y la de un espacio complejo de sociabilidad.

\section{[150] Palabras clave}

Relaciones Bilaterales; Fronteras; Comunicación Política; Análisis del Discurso; Chile; Perú.

Fecha de recepción: junio de 2019

- $\quad$ Fecha de aprobación: noviembre de 2019

\footnotetext{
* Esta investigación ha sido posible gracias al Proyecto Fondo Nacional de Desarrollo Científico y Tecnológico (Fondecyt) Regular 1150812, La regionalización transfronteriza en el norte de Chile, liderado por el doctor Haroldo Dilla y financiado por la Comisión Nacional de Investigación Científica y Tecnológica (Conicyt). Agradecemos el apoyo de las asistentes de investigación Sofía Larrazabal e Ivana Navarro.

** Magíster en Estudios Sociales y Políticos Latinoamericanos. Doctorando en Estudios Americanos. Tesista del Instituto de Estudios Avanzados, Universidad de Santiago de Chile. Correo electrónico: danielbello.a@gmail.com - Orcid: 0000-0003-3636-4618 - Google Scholar: https://scholar.google.es/ citations?user $=2 \mathrm{v} 7$ AtkIAAAAJ\&hl $=\mathrm{es}$

*** Doctor en Filosofía y Letras. Profesor e investigador de la Universidad Tecnológica Metropolitana, Chile. Correo electrónico: chiuminatto@gmail.com - Orcid: 0000-0001-6723-6825 - Google Scholar: https://scholar.google.com/citations?user=G6QNEYkAAAAJ\&hl=en
} 


\title{
Cómo citar este artículo
}

Bello Arellano, Daniel y Chiuminatto Orrego, Magglio Gino. (2020). De línea en el desierto a espacio de sociabilidad. El discurso informativo de la prensa chilena sobre la frontera entre Chile y Perú (2014-2015). Estudios Políticos (Universidad de Antioquia), 57, pp. 150-173. DOI: 10.17533/udea.espo.n57a08

\section{From a Desert Line to a Space of Sociability. The Chilean Press' Informative Discourse on the Border Between Chile and Peru (2014-2015)}

\begin{abstract}
This article analyzes the Chilean press' informative discourse on the border between Chile and Peru, to identify and compare the various narrative proposals that intervene in the symbolic process of constructing the border reality. This analysis seeks, on the one hand, to contribute to the study of a territory that is strained by long-standing bilateral disputes, which is constantly created and re-signified in (and by) public discourse; on the other hand, to compare the role of national and local media in this creative process. The work resulted in the identification of two different ways of understanding and narrating the border, which may be synthesized in two images: a line in the desert and a complex space for sociability.
\end{abstract}

\section{Keywords}

Bilateral Relations; Boundaries; Political Communication; Discourse Analysis; Chile; Peru. 


\section{Introducción}

La relación entre Chile y Perú ha estado marcada, durante buena parte de su historia, por tensiones y disputas territoriales. La Ilamada Guerra del Pacífico, que enfrentó en el último cuarto del siglo xIx a peruanos y bolivianos de un lado, y a chilenos del otro, produjo heridas que, habiendo pasado más de 130 años, no terminan de cicatrizar. Dicha guerra, como suele ocurrir, modificó los territorios de los países involucrados y creó nuevas fronteras que continúan siendo disputadas, redibujadas, resignificadas y, por cierto, vivenciadas.

Las fronteras suelen tener esta cualidad cambiante, polisémica y paradójica. Ellas demarcan el espacio dentro del cual los Estados modernos ejercen soberanía y, por lo mismo, son territorios en los que la soberanía se reafirma y se cuestiona, las identidades nacionales cristalizan y se diluyen, y las fricciones interestatales conviven con relaciones humanas transfronterizas complejas que incluyen relaciones de amistad y cooperación en un marco en constante evolución, tanto en términos político-geográficos como narrativos y semánticos (Bello, 2012a; Cairo y Lois, 2014; Moog, 1957; Nweihed, 1992; Turner, 1987).

En la actualidad, las fronteras reaparecen en el debate público en Chile, Perú y en buena parte del mundo, principalmente en forma de muros, de barreras de control y contención, pero también como escollos físicos y mentales que obstaculizan la integración y el desarrollo de las regiones limítrofes. Esto muestra que son «espacios relativizados [que] para algunos se vuelven invisibles, para otros se mantienen, se trasladan o se refuerzan» (Brenna, 2011, p. 12), que su significado cambia con el tiempo y según la perspectiva de quien la observa — de lejos o de cerca, desde la geopolítica clásica o la crítica, por ejemplo-.

En este sentido, toda frontera es una «categoría heurística» y no una «realidad ontológica», es decir, una entidad geohistórica en constante transformación, que es moldeada — creada y recreada - por las «prácticas materiales y simbólicas de la sociedad» (Benedetti, 2014, p. 15). Es «un objeto inacabado e inestable. Un objeto constantemente disputado de formas diversas. Un objeto que como producto histórico de la acción humana puede ser [y es] reestructurado y resignificado» (Grimson, 2003, p. 17). 
Por ello, este ha sido siempre un eje de tensiones dramáticas, un lugar soñado, imaginado, descrito, narrado, pintado - e investigado- de distintas maneras, la mayor parte del tiempo destacando una u otra de sus múltiples facetas y desconociendo o invisibilizando todas las demás.

Al observar la historia de América Latina, las fronteras pasaron de ser las tenues divisiones de los territorios conquistados y administrados por españoles y portugueses, a ser, luego de las independencias $-\mathrm{y}$ del Uti Possidetis luris de 1810-, los confines de los nacientes Estados. Confines que fueron clarificándose o reconfigurándose — principalmente durante los siglos XIX y $\mathrm{xx}$ - por medio de guerras y la firma de tratados, hasta llegar al actual contexto de líneas dibujadas con cierta precisión. Un diseño que traduce el estado de las fuerzas en contienda al momento de ser definido y que, por lo mismo, no es igualmente aceptado por todas las partes (Bailly, 2013, p. 12).

Por un lado, la relación entre fuertes y débiles, vencedores y vencidos, ha cambiado en los últimos 130 años, por lo que no es de extrañar que, actualmente, quienes se sienten perjudicados cuestionen acuerdos que se tomaron en contextos para ellos desfavorables. Por el otro, la ambigüedad del lenguaje de algunos de los pasajes de los tratados que definieron aquel diseño — varias décadas atrás - y la imposibilidad técnica de la época de delinear con la precisión actual los contornos de los Estados nacionales, ha dejado un margen para la interpretación que ha sido aprovechado por los vencidos - o débiles de entonces - para impugnar partes puntuales de los tratados mencionados (Bello, 2012b).

Esto es lo que apreciamos en el caso de la actual frontera entre Chile y Perú, la cual nació con la Guerra del Pacífico (1879-1883) y la firma del Tratado de Lima de 1929 y que sigue siendo disputada y recreada, legal y narrativamente. Prueba de ello es que en 2008 Perú presentó una demanda contra Chile en la Corte Internacional de Justicia (CIJ) para resolver una controversia por el límite marítimo, la cual se zanjó con el fallo de la disputa marítima Perú v. Chile del 27 de enero de 2014. Inmediatamente después surgió una polémica, esta vez sobre el inicio de la frontera terrestre y el denominado triángulo terrestre, que tiene su origen en las distintas interpretaciones que ambos países hacen del Tratado de 1929. Dicha polémica se intensificó con la creación en noviembre de 2015 del distrito peruano La Yarada-Los Palos (Ley 30358 del 8 de noviembre de 2015), territorio administrativo ubicado en el departamento limítrofe de Tacna y que incluye 3,7 hectáreas —el triángulo mencionado_que ambos países reclaman como propio. 
Es importante señalar que aquellas querellas interestatales solo representan una de las múltiples dimensiones de este territorio fronterizo que es de los más dinámicos y transitados de la región.

El puesto fronterizo de Chacalluta puede considerarse como uno de los más transitados por personas en el continente. En 2017 [según las estadísticas de Aduana, 2017], se produjeron 3297847 ingresos de personas a territorio chileno por este punto, y salieron por él 3229 959, es decir, más de seis millones y medio de cruces anuales. Más del $95 \%$ de estas personas eran chilenas o peruanas, casi a partes iguales (Dilla, 2018, p. 9).

Este territorio fronterizo es uno de los pocos lugares de la extensa geografía de Chile en que una ciudad de regular tamaño de un país vecino - la peruana Tacna - se encuentra íntimamente conectada a una chilena — la ciudad de Arica- (Dilla, 2018). Y, sin embargo, son las querellas las que se resaltan, las que tiñen el discurso público — en especial las heterorrepresentacionesacerca de la frontera, y las que prevalecen en la percepción ciudadana de lo que allí acontece.

En este sentido, y particularmente en escenarios como el descrito, [154] los medios de comunicación desempeñan —lo han hecho siempre- un rol fundamental, ya que no solo entregan información a la población, sino que crean y divulgan representaciones discursivas que canalizan formas de entender y de narrar la frontera y lo fronterizo. Como afirma Silvia Gutiérrez (2010) — siguiendo la argumentación de Miquel Rodrigo Alsina (2005)—:

Una primera característica del discurso de los medios de comunicación es que éstos construyen una representación de la realidad a la que aluden. Sin embargo, esa realidad que refieren es una realidad compleja, diversa y cambiante; es una realidad social poliédrica de la que sólo damos cuenta de algunas de sus caras (p. 171).

Tales representaciones no son meras descripciones objetivas e inocuas. Son, por el contrario, elementos — parciales - constitutivos de la realidad que tienen incidencia sobre las percepciones sociales, las políticas aplicadas al territorio, las posibilidades de desarrollo regional $y$, en definitiva, sobre las vidas de quienes habitan y transitan la frontera.

El objetivo de esta investigación es contribuir al estudio de las relaciones fronterizas y de las relaciones internacionales desde una perspectiva particular, la cual consiste en analizar la dimensión significativa, es decir, la producción 
de sentido que se genera en torno al hecho social, jurídico y político que constituye la frontera. En este artículo se argumenta que, para entender la frontera, para llegar a su significado, es fundamental conocer su historia, estudiar sus dinámicas sociales y sus características físicas y geográficas; y es necesario, también, estudiar lo que se dice de ella, especialmente en los discursos puestos en circulación por los medios de comunicación.

Las fronteras tienen materialidad, objetos que pueden ser examinados y que permiten recurrir a una «explicación ostensiva» ${ }^{1}$ de su significado: ríos y cordilleras, hitos a lo largo de la línea limítrofe, faros, puestos de control, y en algunas ocasiones muros, alambradas y campos minados. No obstante, aquellos objetos no poseen un significado estático: Como señala Stuart Hall (2013, p. 45), «ellos solo adquieren significado y se convierten en objetos de conocimiento dentro del discurso» (p. 24. Traducción propia). En este sentido, afirma que «el punto principal es que el significado no es inherente a las cosas, al mundo. Se construye, se produce. Es el resultado de una práctica significativa, una práctica que produce significado, que hace que las cosas signifiquen» (p. 24. Traducción propia).

Son los discursos los que le dan sentido a la «realidad fronteriza», son estos discursos los que ponen en circulación visiones sobre la frontera y nos permiten conocer y compartir una mirada —la hegemónica - acerca de sus características y de lo que allí ocurre. En consecuencia, «investigar una frontera exige pensar cómo es cotidianamente producida, recreada y reproducida por los diferentes agentes sociales que intervienen en ese espacio» (Cerbino, Ramos y Rodríguez, 2015, p. 253). Y, probablemente, no hay agentes con más capacidad de incidencia en estos procesos creativos que los medios de comunicación, los cuales cumplen una función central en la elaboración y transmisión de las prácticas simbólicas y la noticia —en particular — es «un marco a través del cual se construye rutinariamente el mundo social» (Van Dijk, 1990, p. 22).

Este trabajo se propone estudiar la forma en que la frontera chilenoperuana es presentada, contada, contextualizada y recreada por el discurso

\footnotetext{
${ }^{1}$ Las explicaciones ostensivas son las que recurren a elementos del entorno para dar con el significado de lo que se quiere definir. Sin embargo, argumenta Ludwig Wittgenstein (1976), el significado no está en el objeto al que señalamos en la explicación ostensiva, tampoco en la imagen formada en la mente a partir de una palabra o frase, ni en las palabras que surgen en las explicaciones verbales. El significado de una palabra o una frase, y el sentido de una proposición - matemática, por ejemplo- está en su uso y en su relación con otras palabras o signos en el marco del sistema de signos —el lenguaje - al que pertenece.
} 
informativo de los medios escritos. Para ello, y para contrastar las diversas propuestas narrativas que dan cuenta del enfrentamiento entre autopercepciones -locales - y heteropercepciones -nacionales - (Valenzuela, 2000), se analizaron 295 noticias acerca de la frontera publicadas por dos de los periódicos de circulación nacional más relevantes del país - El Mercurio y La Tercera- y por dos medios digitales de la región fronteriza - El Morrocotudo y Arica al Día- entre el 20 de enero de 2014 — una semana antes del fallo de la Corte Internacional de Justicia por la disputa marítima entre Chile y Perú- y el 14 de noviembre de 2015 —una semana después de la promulgación de la ley que creó el distrito peruano La Yarada-Los Palos-. De esta manera, se busca contribuir al estudio de la frontera chileno-peruana y también poner en cuestión el rol de los medios de comunicación en la construcción social de la realidad fronteriza.

\section{Metodología}

Este trabajo concentra la atención en el proceso de producción de sentido sobre el fenómeno social de la frontera y en los medios de comunicación, entendidos estos como actores institucionales —empresas sujetas a intereses comerciales vinculadas a redes de poder económico y

[156] político- que cumplen un papel protagónico en la distribución de sentido tanto en el ámbito nacional como local. Se observó, desde la perspectiva de análisis del discurso, el proceso de construcción (Potter, 1998) que expresan materialmente estas empresas periodísticas, a través del lenguaje y el texto. Se analizaron los materiales y las herramientas utilizadas para construir la frontera en su dimensión simbólica, y se exploró el texto en tanto instrumento para descubrir algo más (Halliday y Matthiessen, 2004). Como argumenta Michel Foucault (1979, p. 81), «es indudable que los discursos están formados por signos; pero lo que hacen es más que utilizar esos signos para indicar cosas. Es ese más lo que los vuelve irreductibles a la lengua y a la palabra. Es ese "más" lo que hay que revelar y hay que describir».

Teun van Dijk (1990, p. 41) señala que el principal objetivo del análisis del discurso «consiste en producir descripciones explícitas y sistemáticas de unidades del uso del lenguaje al que hemos denominado discurso». No obstante, es imposible — para este estudio como para prácticamente cualquier otro- abordar una descripción explícita y sistemática de todos los aspectos y niveles del texto que conforma el corpus. En este sentido, esta investigación se concentra en enunciados publicados por cuatro medios escritos, sujetos a un contexto específico y a un campo asociado, dentro del cual los tratados y las 
declaraciones oficiales ocupan una posición predominante, a partir de lo cual se construye la referencialidad y el correlato de la frontera (Foucault, 1979).

La exploración del texto estuvo guiada por las siguientes preguntas: ¿cuáles son las características del discurso nacional y local?, ¿son coincidentes en su proceso de construcción, en sus materiales y herramientas, o tocan cuerdas distintas de la multiplicidad de sentidos que se entrelazan en torno a la frontera?

Apoyados por un programa para análisis cualitativo de datos (Atlas.ti, versión 7), se seleccionaron las cláusulas del texto donde estaba presente el término «frontera», para lo cual se consideró la extensión de frases delimitadas por un punto seguido o párrafos delimitados por un punto y aparte. Dentro de estas cláusulas se analizó la colocación (Halliday y Matthiessen, 2004, p. 38), es decir, el grado en que la ocurrencia de una palabra aumenta en presencia de otra, en este caso, el grado en que la ocurrencia de ciertas palabras aumenta ante la presencia de la palabra «frontera». Sin embargo, no se consideró únicamente la posibilidad de ocurrencia de ciertas palabras asociadas al término «frontera» —nodo- dentro de la cláusula, sino también la ocurrencia de temas y acciones asociados al concepto de frontera. De tal manera, fue posible determinar categorías vinculadas al tratamiento del tema dentro del corpus seleccionado.

Para ascender semánticamente en la estructura del texto, desde las palabras a las proposiciones que componen la estructura semántica de categorías asociadas al término «frontera», se utilizaron las macrorreglas descritas por Van Dijk (1990), referentes a procesos de generalización y construcción conceptual, y a la supresión de datos prescindibles. De esta manera, se pudieron establecer categorías temáticas asociadas al tratamiento informativo de la frontera, las cuales fueron empleadas para la codificación del corpus. Así, la colocación de elementos semánticos como Hito 1, Punto Concordia, Tratado de 1929 o Corte Internacional de Justicia en la misma cláusula, la cual incluye al término «frontera», fue categorizada, por construcción, bajo el concepto «Hecho Jurídico». La colocación semántica del comercio, el turismo, el narcotráfico, la inmigración —legal e ilegal—, junto al término «frontera», los cuales remiten a la vida y complejidades que se experimentan en esta parte del territorio, fue categorizada, también por construcción, bajo el concepto «Hecho Social». ${ }^{2}$

${ }^{2}$ Es importante señalar que estas son categorías emergentes, no teóricas, que surgen de las propias
fuentes - en una revisión preliminar - y que son establecidas utilizando las macrorreglas desarrolladas 
Por otra parte, se analizó también lo que denomina sujetos lógicos (Halliday y Matthiessen, 2004), es decir, los actores, a quienes se atribuye la declaración o referencia acerca de la frontera, y que coinciden, en términos generales, con las fuentes utilizadas por los medios periodísticos para construir sus informaciones. El actor o sujeto lógico es quien entrega a la cláusula su carácter de «representación», es decir, una porción o cantidad de experiencia humana expresada a través de las palabras. En otras palabras: «la cláusula, en su función representacional, construye un quantum de experiencia humana: algunos procesos, algunos cambios o, en el límite, ausencia de cambio, en el ambiente externo o en nuestro propio interior» (p. 79. Traducción propia).

La utilización de actores —en rol de fuentes- como el canciller, ministros y presidente fue agrupada, por generalización, bajo la categoría «Autoridades Nacionales»; actores como los alcaldes, concejales y secretarios regionales ministeriales fueron agrupados, por generalización, como «Autoridades Locales»; e iniciativas y representantes de la sociedad civil, como agrupaciones culturales y religiosas, pescadores, comerciantes y agricultores, fueron categorizadas, por construcción, como «Ciudadanía». ${ }^{3}$

«Formalmente hablando, las macrorreglas son reglas de proyección semántica o transformaciones que relacionan proposiciones de nivel más bajo con macroproposiciones de nivel más alto; esto significa que los asuntos o temas derivan de los significados de un texto a través de este resumen de macrorreglas» (Van Dijk, 1990, p. 56). Estos asuntos y temas dan cuenta de la macroestructura semántica del corpus.

\subsection{Corpus}

El corpus de este análisis está compuesto por 295 noticias — documentos primarios- publicadas en Chile entre el 20 de enero de 2014, una semana antes del fallo de la Corte Internacional de Justicia por la disputa marítima entre Chile y Perú, y el 14 de noviembre de 2015, una semana después de la promulgación de la ley que creó el distrito peruano La Yarada-Los Palos.

por Van Dijk (1990). Aquellas son «reglas que se aplican al conjunto de proposiciones que forman un texto para obtener su macroestructura, entendida esta como el contenido semántico que resume el sentido del texto» (Centro Virtual Cervantes, s. f.).

${ }^{3}$ La supresión consiste en eliminar la información que no sea relevante para el significado general del texto. La generalización reemplaza una serie de términos por un término de significado más amplio que los engloba. Y la construcción genera un concepto que vincula semánticamente una serie de términos que cuentan con determinados aspectos en común. 
Las noticias seleccionadas provienen de cuatro medios, dos de circulación nacional y dos regionales, que fueron elegidos por su relevancia e idoneidad para los efectos de esta investigación. Los dos medios nacionales, La Tercera y El Mercurio, son los más tradicionales e importantes del país, y pertenecen a dos grupos de medios - Grupo Copesa y Grupo El Mercurio, respectivamente- que concentran cerca de $90 \%$ de los diarios y de los lectores en Chile (Poderopedia, 2015, septiembre 9).

Los dos diarios regionales, El Morrocotudo y Arica al Día, ambos electrónicos y con base en la ciudad de Arica, tienen una indiscutida importancia local y no pertenecen al duopolio Copesa-El Mercurio, por lo que tienen una línea editorial independiente que facilita la tarea de contrastar los discursos informativos nacionales y regionales.

Las noticias fueron seleccionadas luego de revisar exhaustivamente, tanto en las ediciones digitales como en las versiones impresas de los medios antes señalados, todo el contenido en el que se mencionara la palabra frontera -nodo- . Se descartaron las columnas de opinión, las notas no informativas y todos los artículos que no tuvieran relación directa y explícita con el tratamiento noticioso de la frontera chileno-peruana.

Como resultado, se obtuvieron 134 noticias de El Mercurio, 81 de La Tercera, 63 de El Morrocotudo y 17 de Arica al Día. La diferencia entre la cantidad de noticias encontradas y seleccionadas en los diarios nacionales y regionales -215 frente a 80 - se debe al menor número de noticias publicadas y almacenadas en los sitios web de estos últimos, y a la menor precisión de sus motores de búsqueda.

Tabla 1. Composición del corpus.

\begin{tabular}{|l|c|c|c|}
\hline \multicolumn{1}{|c|}{ Diarios } & Nacionales & Regionales & Total \\
\hline El Mercurio & 134 & & \\
\hline La Tercera & 81 & & \\
\hline El Morrocotudo & & 63 & \\
\hline Arica al Día & & 17 & \\
\hline Total & $\mathbf{2 1 5}$ & $\mathbf{8 0}$ & $\mathbf{2 9 5}$ \\
\hline
\end{tabular}

Fuente: elaboración propia. 


\section{Análisis del discurso informativo sobre la frontera entre Chile y Perú}

Para llevar a cabo el análisis, se identificaron las cláusulas específicas en las que estaba presente el término «frontera» en los 295 documentos primarios. Esto dio como resultado 747 cláusulas. En ellas se detectaron coocurrencias relevantes con las categorías temáticas «Hecho jurídico» — 942 ocurrencias- y «Hecho social» - 345 ocurrencias-, y con actores a quienes se entrega la posibilidad de pronunciarse sobre este tema: «Autoridades nacionales» — 1340 ocurrencias—, «Autoridades locales» — 221 ocurrencias—, «Ciudadanía» - 247 ocurrencias—. ${ }^{4}$ De este modo, se pudo reconocer algunas características del discurso informativo sobre la frontera y diferencias significativas entre los discursos nacionales y regionales.

\subsection{Una línea en el desierto: la frontera en el discurso de los medios nacionales}

El primer hallazgo relevante es el tono monocromático del relato construido por los medios nacionales. Una narrativa (Bruner, 1991) sobre la frontera que minimiza la historicidad humana, donde prácticamente no

[160] existe el tránsito de personas, la construcción de lazos ni el comercio, y donde tampoco existen —salvo raras excepciones - las ciudades fronterizas de Tacna y Arica. En este relato, la frontera es una definición legal, carente de habitantes y territorio, instaurada únicamente por el Tratado de Lima de 1929. Como señala Jerome Bruner (1991, p. 4), «nosotros organizamos nuestra experiencia y nuestra memoria de los sucesos humanos principalmente en la forma narrativa: historias, excusas, mitos, razones para hacer y para no hacer, etcétera» (traducción propia). Y, en este caso, los elementos utilizados para construir la narrativa nacional sobre la frontera, son extremadamente reducidos.

Los referentes urbanos y geográficos son remplazados por coordenadas asépticas como Hito 1, que remiten a ese momento fundacional que parece haber fijado un significado esencial de la frontera —en tanto línea limítrofe-,

\footnotetext{
${ }^{4}$ El hecho de que una categoría tenga un número de ocurrencias mayor al total de cláusulas se explica porque sus componentes semánticos están presentes, en promedio, más de una vez por cláusula, por ejemplo: ministros, presidentes, cancilleres, miembros de las comisiones de relaciones internacionales del Congreso, en el caso de «Autoridades Nacionales».
} 
que solo puede ser puesto en cuestión por nuevas definiciones jurídicas: acuerdos interestatales y fallos de cortes internacionales. La frontera se muestra como objeto de una disputa internacional, que se desarrolla lejos de la zona fronteriza, principalmente en la CIJ de La Haya, Países Bajos, y en las capitales Santiago, Chile, y Lima, Perú. Una disputa que está marcada por acontecimientos puntuales como el fallo de la Corte Internacional de Justicia, la emergencia del conflicto limítrofe por el triángulo terrestre y la creación del distrito peruano La Yarada-Los Palos, los cuales refuerzan la idea de que la frontera es, en esencia, una línea y un hecho jurídico que separa a dos entidades soberanas.

Una parte muy importante de la información presentada por los medios nacionales se dedica a tratar estos temas, dejando de lado otros asuntos, no menos importantes, como los efectos sociales del conflicto bilateral. Muestra de lo anterior son estas citas extraídas de El Mercurio y La Tercera:

El presidente Sebastián Piñera eludió confirmar el envío de una nota de protesta a Perú por las diferencias surgidas sobre la soberanía del «triángulo terrestre» en la frontera, tras el fallo de la Corte Internacional de Justicia (CIJ) de La Haya, pero sí dijo estar convencido de que «es chileno» (El Mercurio, 2014, febrero 10).

El canciller chileno, Heraldo Muñoz, informó el martes de que había enviado una nota diplomática a Perú, luego de que el Parlamento peruano aprobara el proyecto que crea el distrito de La Yarada Los Palos, que incluye un punto de la frontera terrestre que ambos países consideran parte de su territorio (La Tercera, 2015, octubre 22).

Esta apreciación queda de manifiesto al medir las ocurrencias de las categorías temáticas en la prensa nacional. Como se observa en la gráfica 1, de las 1022 veces en que estos diarios hacen alusión a la frontera como «Hecho Social» o «Hecho Jurídico», 817 veces (80\%) lo hacen refiriéndose a hechos puramente jurídicos - a los hitos, al Punto Concordia, al Tratado de 1929, a los tratados de 1952 y 1954, a la Corte Internacional de Justicia, entre otros- , sin considerar el espacio geográfico, la historia del territorio ni a la población que lo habita. Y solo 205 veces (20\%) los medios nacionales establecen una relación entre la frontera y conceptos que dan cuenta de un espacio de sociabilidad, que se habita y transita, y en el que se producen intercambios, comercio, diálogos, encuentros y también fricciones. 
Gráfica 1. Categorías temáticas en la prensa nacional, ocurrencias y porcentaje.

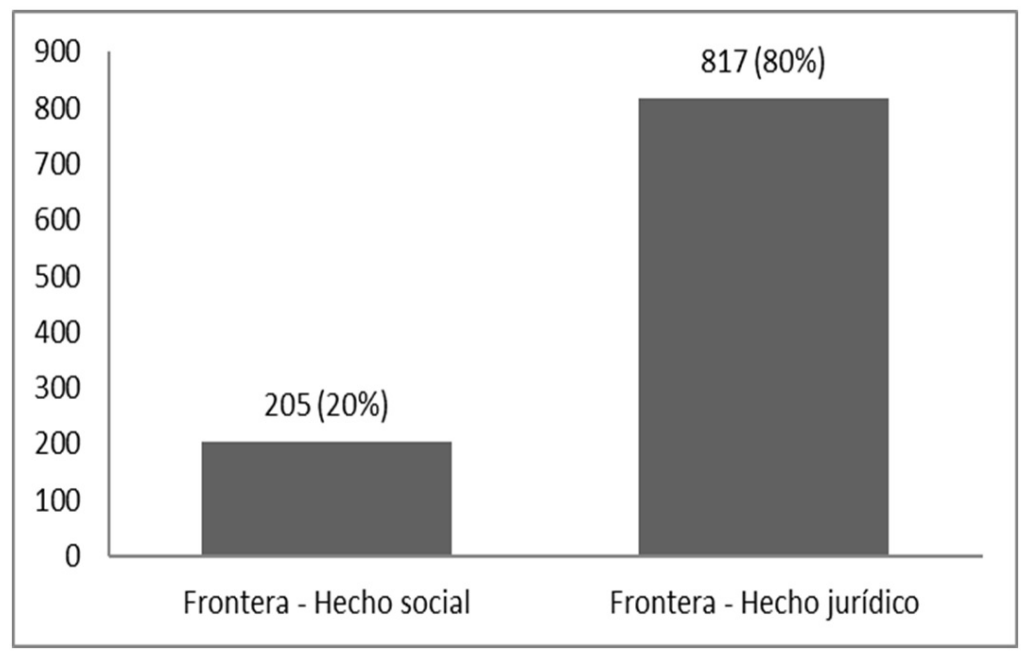

Fuente: elaboración propia.

Los casos excepcionales en que la prensa nacional establece una relación entre la frontera y la categoría «Hecho social» suelen estar marcados por acontecimientos policiales y por informaciones puntuales sobre el flujo de migrantes, turistas y comerciantes entre las ciudades de Arica y Tacna, por ejemplo:

Hasta las 20:00 horas de ayer, poco más de 3.500 personas habían cruzado la frontera norte en dirección a Perú por el paso Chacalluta, ubicado a $20 \mathrm{~km}$ de Arica. Un flujo que solo corresponde al 35\% de la proyección diaria que había hecho el Servicio Nacional de Aduanas para este fin de semana largo de Fiestas Patrias, en el control que, con más de 5,5 millones de trámites migratorios al año, es el que registra mayor flujo de personas en todo el país (El Mercurio, 2015, septiembre 19).

Por otra parte, el relato de los medios nacionales sobre la frontera se construye con muy pocos actores, que «reafirman», «reiteran», «insisten» sobre sus mismas declaraciones acerca del trazado establecido e «intangible» de la frontera, creada por el Tratado de 1929 y materializada por los hitos -el Hito 1 en particular - colocados a lo largo de la línea limítrofe. En este sentido, a grandes rasgos, la narración constituye un relato mítico, en donde un nuevo orden de cosas — un nuevo statu quo — surge de la Guerra del 
Pacífico y se consolida en la acción creadora del Tratado, el cual se transforma en la fuente de la verdad sobre la frontera chileno-peruana.

Por lo mismo, no hay espacio para interpretaciones que difieran de la oficial — la cual ha sido sacralizada en la práctica - ni tampoco para el entendimiento entre las posiciones oficiales de ambos países. Para los diarios chilenos de circulación nacional, la frontera es lo que los intérpretes autorizados del Tratado de 1929 — las fuentes oficiales - dicen que es: una línea y una barrera instaurada en el desierto, cuyo punto inicial es el «mítico» Hito 1. Un buen ejemplo de esto es la declaración del entonces ministro de defensa chileno, Jorge Burgos, refiriéndose a la frontera y a la controversia con Perú por el triángulo terrestre:

[El ministro] reiteró que «la frontera comienza en el Hito 1 y no hay ningún triángulo, ese es un artilugio», agregando que «Chile tiene la absoluta convicción jurídica de que eso es así. Si hay otro país que cree que jurídicamente no corresponde, allá ellos» (EI Mercurio, 2014, septiembre 1).

No extraña, por tanto, que los medios nacionales recurran en forma casi exclusiva a las fuentes oficiales vinculadas al gobierno central y al Congreso, y dentro de ellas a pocas, fundamentalmente los presidentes, cancilleres, ministros del Interior y de Defensa, y a parlamentarios de comisiones de Relaciones Exteriores. Estas fuentes, además, utilizan un repertorio reducido de argumentos, básicamente legales, que se remiten al Tratado de 1929 y a otros acuerdos firmados por Chile y Perú. Clara muestra de lo anterior es la opinión del entonces ministro de Relaciones Exteriores de Chile:

[El canciller Heraldo Muñoz] recalcó que no entrarán en un debate público sobre esta materia [el triángulo terrestre], e insistió en que «nuestros derechos están muy claramente consignados en el Tratado de 1929, en los trabajos de las comisiones en 1930 particularmente, de modo que en eso no hay duda y tenemos total tranquilidad respecto a los derechos que nos asisten» (Romero, 2014, agosto 28).

Al objetivar los datos, contando las ocurrencias de los sujetos lógicos -actores-, de las 1414 veces que los medios nacionales dan voz a algún actor para que se refiera a la frontera, 1197 veces (85\%) la voz pertenece a una autoridad del Gobierno central, 79 veces (5\%) a una autoridad local y 138 veces $(10 \%)$ a la ciudadanía — diversos actores de la sociedad civil—. 
Gráfica 2. Voces recogidas por la prensa nacional, ocurrencias y porcentaje.

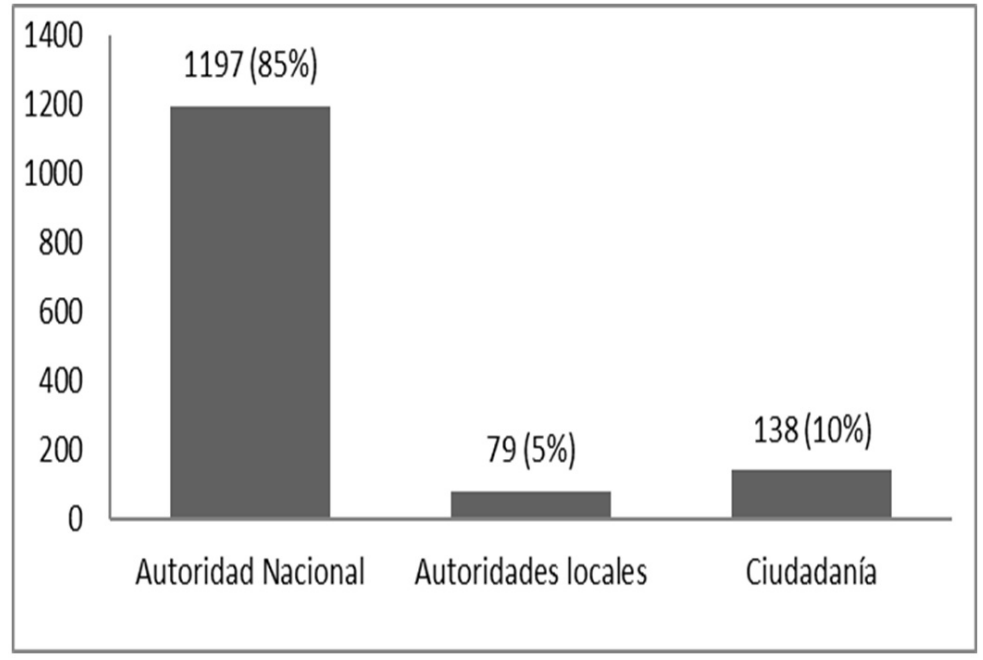

Fuente: elaboración propia.

Se opera, de esta manera, una escala de reducciones, en primer lugar, de las fuentes posibles, donde las fuentes oficiales nacionales ocupan un lugar sin contrapeso; y de la narrativa, de los argumentos y temáticas en torno a la frontera. El correlato que construyen estos enunciados, el «conjunto de dominios en los que tales objetos pueden aparecer y en los que tales relaciones pueden ser asignadas» (Foucault, 1979, p. 51) está marcado y delimitado absolutamente por la hoja del Tratado. Cualquier modificación de esta instauración legal de la frontera es vista como una amenaza capaz de quebrar el orden establecido. Así, el duelo de declaraciones se articula como una defensa de lo estable y zanjado, frente a lo negociable e indeterminado que conduce al conflicto.

De este modo, la trama informativa, en su simpleza y reiteración, lucha contra la posibilidad de caos y desintegración simbólica contra la posible alteración de la frontera, en detrimento de la soberanía y el «interés nacional» de Chile, descartando de plano los intereses de la sociedad fronteriza y las voces de las personas — comerciantes, pescadores, agricultores y transportistasque habitan aquel territorio. La limitación de recursos dramáticos, en este sentido, no permite ampliar el imaginario ni el conocimiento en el ámbito nacional sobre lo que acontece en la frontera. 
En el discurso de los medios nacionales, la frontera es presentada como un hecho jurídico, una línea descrita en un tratado que atraviesa un desierto real, el más árido del mundo, y la cual está inmersa en un desierto narrativo.

\subsection{La frontera habitada y viva de los medios regionales}

Al comparar la construcción informativa de los diarios nacionales con el discurso generado por los medios regionales, la textura de la frontera se despliega en sus diversos aspectos como espacio vivo, rico en intercambios comerciales y con problemas prácticos asociados a su permeabilidad — como los que enfrentan los agricultores y los pescadores-, y aquellos relacionados con los pasos ilegales, el narcotráfico y la caza furtiva. Se visualizan, por otra parte, las iniciativas que surgen entre sus habitantes e instituciones locales para promover la integración, tanto en el plano económico como en el cultural y sanitario. La frontera aparece como un espacio de encuentro social y no solo como un asunto en disputa entre Estados.

Mientras el discurso nacional se caracteriza por su intento de asegurar el límite, el discurso local destaca la vida junto al otro, el cual cohabita el territorio transfronterizo. Esta es, a grandes rasgos, la distinción entre una heterorrepresentación centralista que ve la frontera como final y vuelca la mirada hacia adentro, hacia el lugar desde donde se ejerce el poder —la capital- , y una autorrepresentación local que, por el contrario, mira hacia afuera y ve el comienzo de un espacio geográfico, económico y social compartido. Por ejemplo:

La actividad denominada «Encuentro binacional de dirigentes sociales por la paz» congregó a más de 200 personas, quienes en representación de cada ciudad caminaron desde sus respectivas fronteras hasta juntarse en la línea que divide a ambos países, pero que no es capaz de dividir el espíritu de sana convivencia que se proyectan al futuro [sic] unidos más que nunca (El Morrocotudo, 2014, julio 11).

Por ello, a partir de la información regional, el tema de la frontera no es su trazado sino su tránsito, su permeabilidad y su control. Los actores locales expresan la necesidad de establecer dinámicas más efectivas de intercambio $y$, al mismo tiempo, de vigilancia, para detener el ingreso de droga y la inmigración ilegal, como medios para avanzar hacia el desarrollo de la región. Esto se observa, por ejemplo, en la siguiente referencia al trabajo del Comité de Integración y Desarrollo Fronterizo Chile-Perú: 
El intendente Emilio Rodríguez manifestó que esta instancia binacional permite no sólo afianzar el trabajo en los complejos aduaneros, sino que potencia la integración entre los dos países y, especialmente, entre Arica y Tacna. «Esta integración busca trabajos de colaboración y participación mutua; por eso es muy importante lo que se realizará durante esos días, avanzando en el control integrado, pero con una mirada de unidad, donde la sinergia, la complementación económica, productiva y social forma parte de la mirada de desarrollo de los dos países», comentó (El Morrocotudo, 2014, septiembre 4).

Al hacer el ejercicio de contar las coocurrencias de las categorías temáticas en la prensa local, se observa con toda claridad el contraste con lo que sucede en los medios nacionales. En este caso, las menciones a la frontera están más relacionadas con «Hechos Sociales» que con «Hechos Jurídicos». De las 265 veces en que estos medios se refieren a tales categorías, 140 veces (53\%) lo hacen aludiendo a algún «hecho social», es decir, a conceptos como intercambio, comercio, encuentro, diálogo, integración y otros similares que denotan relaciones humanas y que «construyen» la frontera como un espacio de sociabilidad. En tanto, 125 veces (47\%) lo hacen refiriéndose a la categoría «Hecho Jurídico», esto es, a los tratados de 1929, 1952, 1954, al Hito 1, al Punto Concordia, entre otros.

Gráfica 3. Categorías temáticas en la prensa regional, ocurrencias y porcentaje.

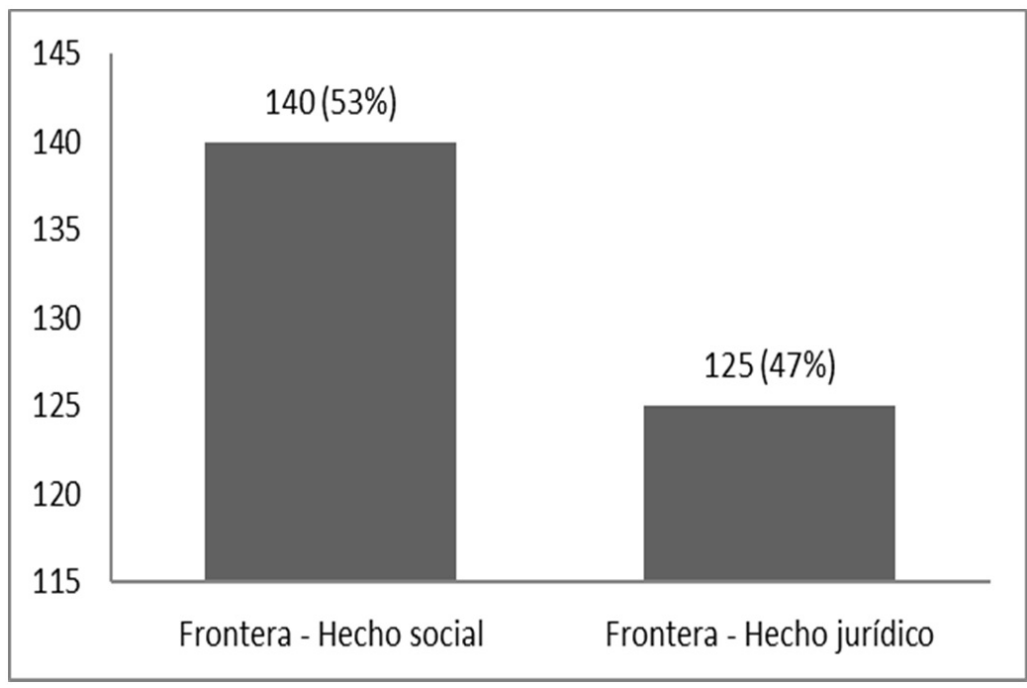

Fuente: elaboración propia. 
Un contraste similar se aprecia cuando medimos las coocurrencias de los sujetos lógicos - actores-. En este caso, de las 394 veces en que los medios locales recurren a una fuente para que hable de la frontera, 143 veces (36\%) la fuente es una autoridad nacional, 142 veces (36\%) una autoridad local y 109 veces (28\%) algún representante de sociedad civil regional.

Gráfica 4. Voces recogidas por la prensa regional, ocurrencias y porcentaje.

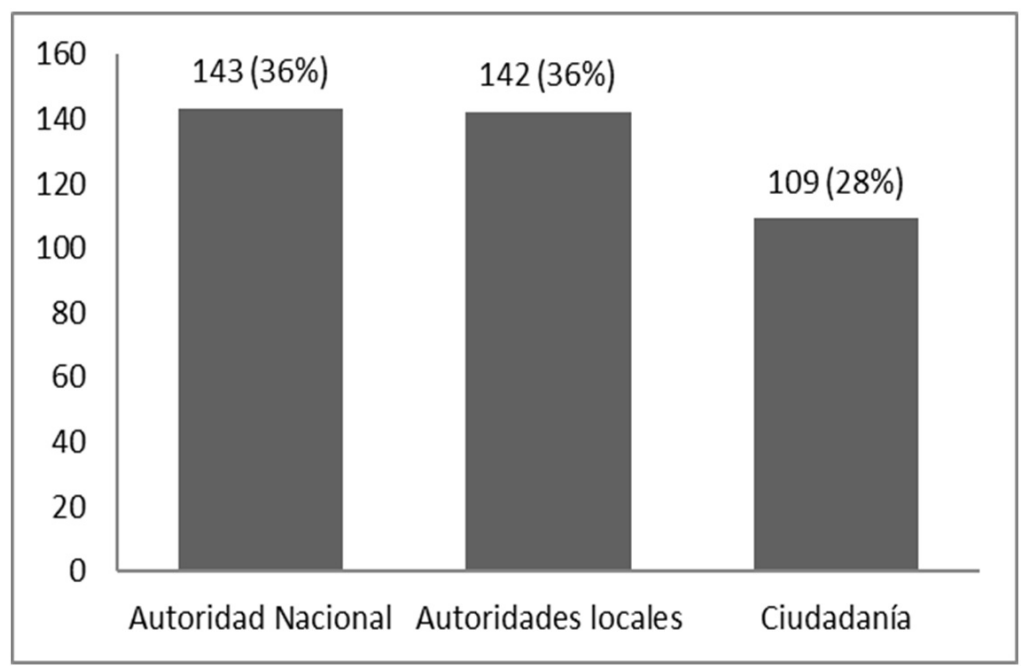

Fuente: elaboración propia.

A partir de estos datos se observa una diversificación mucho mayor de las fuentes, las cuales incorporan a autoridades nacionales, a autoridades locales y a la ciudadanía en una relación prácticamente de paridad como voces autorizadas para referirse a la frontera. Por ejemplo:

El 10 de julio delegaciones vecinales de Arica y Tacna realizarán un simbólico encuentro en la Línea de Concordia para fortalecer los lazos de integración fronteriza. Así lo dio a conocer Eduardo Gutiérrez, presidente de la Unión Comunal de Juntas de Vecinos de Arica «San Marcos», en la cuarta cita de coordinación de la Subcomisión de Desarrollo Social y Género del Comité de Integración y Desarrollo Fronterizo entre Perú y Chile, que sesionó en Tacna (El Morrocotudo, 2014, julio 2). 
Equipos de salud de Arica y Tacna realizarán un simulacro conjunto en la frontera. La seremi de Salud de Arica y Parinacota, Giovanna Calle, explicó que durante los últimos meses los equipos de salud de las regiones de Arica y Parinacota y Tacna, juntos al apoyo de los ministerios de ambos países, han estado trabajando en la planificación de este simulacro que tiene como fin medir la capacidad de respuesta cuando se registre una emergencia que amerite la concurrencia de una asistencia binacional e integrada (El Morrocotudo, 2014, diciembre 13).

El gerente de la Corporación de Desarrollo de Arica y Parinacota, Sergio Giaconi Mozó, entregó al Embajador de Chile en Perú, Roberto Ibarra García, el documento «Arica y Parinacota, Región de Integración Chile-Perú», el que contiene - entre otros considerandos- iniciativas tendientes a lograr una mayor y mejor integración entre ambos países (Arica al Día, 2015, agosto 17).

La frontera habitada comercia, migra, comparte. Es anterior, incluso, al hecho jurídico que la fija. Es el territorio que los pueblos altiplánicos han transitado desde mucho antes que surgieran los Estados nacionales. Esta realidad — que los medios nacionales invisibilizan- se refuerza con iniciativas como la Feria Camélida Trinacional que, como dice el alcalde de la comuna chilena de General Lagos, ubicada en la región fronteriza de Arica y Parinacota, «demuestra que el pueblo aymara no tiene fronteras y que los límites son sólo circunstanciales» (El Morrocotudo, 2015, mayo 18). Se pone así en cuestión la noción monolítica del límite establecido jurídicamente y su importancia para el orden internacional.

Esta diversidad de voces, de experiencias y de relatos sobre la frontera contribuye a la construcción, por parte de los medios locales, de una narración compleja que recoge las múltiples dimensiones y sentidos de lo fronterizo. Una narración que, si trascendiera el ámbito local, si tuviera cabida en los medios de comunicación nacionales, podría enriquecer el debate público sobre las necesidades y posibilidades de desarrollo de una zona que, por cierto, tiene vida más allá de los conflictos interestatales e intereses que trascienden las disquisiciones jurídicas y las barreras que separan a chilenos y peruanos.

\section{Conclusiones}

La producción de sentido se expresa en discursos, en circulación de signos, que en este caso se han recopilado de medios de comunicación 
impresos chilenos, de circulación nacional y local, lo cual permite evaluar y comparar al menos dos tipos de discursos informativos, sin pretender agotar con esto el repertorio de discursos existentes, lo que excede con creces las posibilidades de esta investigación. Desde esta perspectiva, se puede evaluar también la contribución que los medios de comunicación hacen al debate público sobre temas de interés general, en este caso, lo que acontece en la frontera entre Chile y Perú.

La primera conclusión es que el discurso acrítico de la prensa nacional, extremadamente limitado en su tratamiento informativo de la frontera, sujeto únicamente a la discusión sobre aspectos jurídicos, no permite una amplitud de visión y elasticidad argumentativa suficientes para superar el enfrentamiento de las posiciones en conflicto. Su utilización prácticamente exclusiva de autoridades nacionales como fuentes periodísticas, sin expertos independientes o visiones contrapuestas de ningún tipo, sin presencia de actores ciudadanos o referencias a la actividad cotidiana en el territorio, no hace más que cerrar el círculo sobre una versión única, sin fisuras, pero, al mismo tiempo, sin posibilidades de encuentro con versiones alternativas.

En este discurso de los medios nacionales acerca de la frontera, los actores son tan pocos y los argumentos tan limitados, que resulta prácticamente imposible construir una narrativa que remita a un correlato más complejo que hable no solo de los conflictos legales que se han suscitado en la definición de un trazado, sino también de las oportunidades y problemas que enfrenta un territorio alejado de la capital — como el fronterizo — para desplegar sus potencialidades y lograr su desarrollo.

La segunda conclusión es que la información local, a diferencia de la nacional, diversifica las temáticas asociadas a la frontera y abre espacios para que ingrese también al discurso informativo la experiencia de la vida cotidiana: el comercio transfronterizo, el turismo, las relaciones culturales y religiosas, las vinculaciones humanas que anteceden a las delimitaciones de los Estados nacionales, así como los problemas que enfrenta esta zona, como son el narcotráfico o la inmigración ilegal, entre otros.

Esta apertura es coherente, además, con la diversificación de fuentes utilizadas, que se distribuyen de manera homogénea entre autoridades nacionales, autoridades locales y ciudadanía. La frontera, de esta forma, deja de ser la palabra jurídica del tratado y la línea abstracta que cruza el desierto, 
para adquirir la materialidad del territorio habitado, con las oportunidades y dificultades propias de la existencia humana.

Sin duda, este discurso más complejo construye versiones menos monolíticas. La estructura rígidamente establecida muestra fisuras y se debilita. Los tratados pasan a ser una versión más entre una serie de versiones posibles. La frontera existe en tanto convención —límite-, pero existe igualmente como espacio de socialización, como continuidad histórica, como tierra de oportunidades, las cuales podrían incrementarse si disminuyeran las divisiones y barreras.

Esta suma de elementos narrativos permite que las historias de uno y otro lado de la frontera se encuentren y se creen espacios de confluencia que hagan posible avanzar hacia la superación del enfrentamiento entre dos Estados - Chile y Perú- que no logran dejar atrás una guerra que tuvo lugar hace más de 130 años. En este sentido, el trabajo periodístico de los medios locales se destaca por su aporte. Las empresas que están detrás de aquella labor cuentan con menores recursos económicos, comparadas con los grandes grupos periodísticos chilenos - Copesa y El Mercurio-, pero realizan un tratamiento informativo más complejo y diverso de la frontera.

[170] Sin embargo, esta narración local es prácticamente ignorada por los grandes medios y, en consecuencia, por la opinión pública nacional.

La función de los medios, en el marco del sistema democrático, es - $\mathrm{O}$ debiera ser- multiplicar los enfoques y miradas sobre los asuntos públicos para enriquecer - y democratizar - los debates, especialmente cuando se trata de temas como el que se abordó en esta investigación, los cuales tienden a presentarse de forma maniquea, toda vez que afectan el «interés nacional», entendido como interés de Estado. Los medios como simples órganos reproductores de un comunicado o versión oficial no colaboran en esta tarea.

\section{Referencias bibliográficas}

1. Arica al Día. (2015, agosto 17). Cordap Entregó Propuestas al Embajador de Chile en Perú. Recuperado de https://www.aricaldia.cl/cordap-entrego-propuestas-alembajador-de-chile-en-peru/

2. Bailly, Antoine. (2013). Las fronteras: representaciones, poderes y divisiones territoriales. En: Núñez, Andrés; Sánchez, Rafael y Arenas, Federico (eds.). Fronteras en movimiento e imaginarios geográficos (pp. 11-14). Santiago: RIL. 
3. Bello, Daniel. (2012a). La Triple Frontera del Paraná (Paraguay-BrasilArgentina). Condiciones endógenas institucionales e ilegalidad. Santiago: RIL.

4. Bello, Daniel. (2012b). Chile y Perú en La Haya: fortalezas, debilidades, paradojas y dudas. El Mostrador. Recuperado de https://www.elmostrador.cl/noticias/ opinion/2012/12/18/chile-y-peru-en-la-haya-fortalezas-debilidades-paradojas-y-dudas/

5. Benedetti, Alejandro. (2014). Espacios fronterizos del sur sudamericano. Propuesta de un modelo conceptual para su estudio. Estudios Fronterizos, 15 (29), pp. 11-47. DOI: 10.21670/ref.2014.29.a01

6. Brenna, Jorge. (2011). La mitología fronteriza: Turner y la modernidad. Estudios Fronterizos, 12 (24), pp. 9-34.

7. Bruner, Jerome. (1991). The Narrative Construction of Reality. Critical Inquiry, 18 (1), pp. 1-21. DOI: 10.1086/448619

8. Cairo, Heriberto y Lois, María. (2014). Geografía política de las disputas de fronteras: cambios y continuidades en los discursos geopolíticos en América Latina (1990-2013). Revista Colombiana de Geografía, 23 (2), pp. 45-67. DOI: 10.15446/ rcdg.v23n2.39578

9. Centro Virtual Cervantes. (s. f.). Macrorreglas textuales. Recuperado de https://cvc.cervantes.es/ensenanza/biblioteca_ele/diccio_ele/diccionario/ macrorreglastextuales.htm

10. Cerbino, Mauro. Ramos, Isabel y Rodríguez, Ana. (2015). Sólo interesados en los muertos. Representaciones mediáticas nacionales y narrativas de los habitantes de la frontera EcuadorColombia. Estudios sobre el Mensaje Periodístico, 21 (1), pp. 251268. DOI: 10.5209/rev_ESMP.2015.v21.n1.49093

11. Corte Internacional de Justicia (CIJ). Disputa marítima. (Peru v. Chile). (27 de enero de 2014). Fallo. Recuperado de https://www.icj-cij.org/files/caserelated/137/137-20140127-JUD-01-00-BI.pdf

12. Dilla, Haroldo. (2018). Arica entre tres fronteras. Estudios Atacameños, 57, pp. 221-238. Recuperado de https://scielo.conicyt.cl/scielo.php?script =sci arttext\&pid = S0718-10432018005000301\&lng = es\&nrm = iso\&tlng =es

13. El Mercurio. (2014, febrero 10). Piñera reafirma que «triángulo terrestre» es chileno: Lo hemos dicho por «varios caminos». Recuperado de http://www.emol. com/noticias/nacional/2014/02/10/644094/chile-envio-nota-de-protesta-al-peru-portriangulo-terrestre.html

14. El Mercurio. (2014, septiembre 1). Ministro de Defensa y pugna con Perú: "Vamos a defender lo nuestro». Recuperado de https://www.emol.com/noticias/ nacional/2014/08/31/677893/ministro-de-defensa-no-tenemos-pretension-deincorporar-de-territorios-que-no-han-sido-nuestros.html

15. El Mercurio. (2015, septiembre 19). Baja afluencia de viajeros tuvo este «18» el paso fronterizo Chacalluta. Recuperado de http://www.economiaynegocios.cl/ noticias/noticias.asp? $\mathrm{id}=184523$ 
16. El Morrocotudo. (2015, mayo 18). Integración entre Chile, Perú y Bolivia, marca séptima jornada de importante feria colaborativa. Recuperado de http://www. elmorrocotudo.cl/noticia/sociedad/integracion-entre-chile-peru-y-bolivia-marcaseptima-jornada-de-importante-feria-co

17. El Morrocotudo. (2014, julio 2). Ciudadanía de Arica y Tacna realizará simbólico encuentro en la Concordia. Recuperado de http://www.elmorrocotudo. cl/noticia/sociedad/ciudadania-de-arica-y-tacna-realizara-simbolico-encuentro-en-laconcordia

18. El Morrocotudo. (2014, julio 11). Líderes de Arica y Tacna se abrazaron en la frontera. Recuperado de http://www.elmorrocotudo.cl/noticia/sociedad/lideres-dearica-y-tacna-se-abrazaron-en-la-frontera

19. El Morrocotudo. (2014, septiembre 4). Arica: Comenzó el tercer Comité de Integración y Desarrollo Fronterizo Chile-Perú. Recuperado de http://m. elmorrocotudo.cl/noticia/listado/arica-comenzo-el-tercer-comite-de-integracion-ydesarrollo-fronterizo-chile-peru

20. El Morrocotudo. (2014, diciembre 13). Equipos de salud de Arica y Tacna realizarán un simulacro conjunto en la frontera. Recuperado de http://m.elmorrocotudo. cl/noticia/listado/equipos-de-salud-de-arica-y-tacna-realizaran-un-simulacro-conjuntoen-la-frontera

21. Foucault, Michel. (1979). La arqueología del saber. México, D. F.: Siglo xxı.

22. Grimson, Alejandro. (2003). Los procesos de fronterización: flujos, redes [172] e historicidad. En: García, Clara Inés (comp.). Fronteras. Territorios y metáforas. Medellín: Hombre Nuevo.

23. Gutiérrez, Silvia. (2010). Discurso periodístico: una propuesta analítica. Comunicación y Sociedad, 14, pp. 169-198.

24. Hall, Stuart. (2013). The Work of Representation. In: Hall, Stuart, Evans, Jessica \& Nixon, Sean (eds.). Representation: Cultural Representations and Signifying Practices. London: Sage.

25. Halliday, Michael \& Matthiessen, Christian. (2004). Introduction to Functional Grammar. New York: Oxford University.

26. La Tercera. (2015, octubre 22). Perú responde que el inicio de su frontera con Chile es el Punto Concordia. Recuperado de https://www.latercera.com/noticia/peruresponde-que-el-inicio-de-su-frontera-con-chile-es-el-punto-concordia/

27. Moog, Vianna. (1957). Bandeirantes e pioneiros: paralelo entre duas culturas. Rio de Janeiro: Globo.

28. Nweihed, Kaldone. (1992). Frontera y límite en su marco mundial. Una aproximación a la «fronterología». Caracas: Equinoccio.

29. Perú. Congreso de la República. Ley 30358. (8 de noviembre de 2015). Ley de creación del distrito de La Yarada Los Palos en la provincia de Tacna del departamento de Tacna. Congreso de la República del Perú. Recuperado de http:// www.leyes.congreso.gob.pe/Documentos/Leyes/30358.pdf 
30. Poderopedia. (2015, septiembre 9). ¿Quiénes son los grandes grupos controladores de medios en Chile? Recuperado de http://apps.poderopedia.org/ mapademedios/analisis/4/

31. Potter, Jonathan. (1998). La representación de la realidad. Discurso, retórica y construcción social. Barcelona: Paidós.

32. Romero, María Cristina. (2014, agosto 28). Canciller Muñoz: «El triángulo terrestre es territorio chileno incuestionablemente». El Mercurio. Recuperado de http:// www.emol.com/noticias/nacional/2014/08/28/677360/canciller.html

33. Turner, Frederick Jackson. (1987). El significado de la frontera en la historia americana. Secuencia, 7, pp. 187-207. DOI: 10.18234/secuencia.v0i07.170

34. Valenzuela, José Manuel. (2000). Norteños ayankados. Discursos y representaciones de la frontera. Comunicación y Sociedad, 38, pp. 37-57.

35. Van Dijk, Teun. (1990). La Noticia como Discurso. Comprensión, estructura y producción de la información. Barcelona: Paidós.

36. Wittgenstein, Ludwig. (1976). Los cuadernos azul y marrón. Madrid: Tecnos. 